REMOTE MANIPULATOR EXPERIENCE IN TARGET TRAIN MAINTENANCE AT FERMILAB

Stephen W. Butala

Radiation Safety officer

Research Division

October 11,1984 
Introduction

Fermilab is a high energy physics research facility located about 35 miles west of Chicago. It operates a four mile circumference superconducting proton synchrotron called the Tevatron, which has recently accelerated protons to an energy of $800 \mathrm{GeV}$ at intensities of up to $1.2 \mathrm{E} 13$ protons/pulse on a 60 second cycle. Prior to Tevatron operation its injection accelerator, called the Main Ring, had operated as the final stage. It typically extracted protons at $400 \mathrm{GeV}$ on a $10 \mathrm{~second}$ cycle with intensities of up to $3 \mathrm{E} 13$ protons/pulse. Protons are transported by electromagnets to three experimental areas and further divided into as many as 12 seperate beamlines. The three areas are known as the Proton, Meson and Neutrino beamline areas. Figure 1 depicts a schematic layout of the Fermilab site as it appeared in 1980 .

The Neutrino Area has used four different configurations of beamline components to target protons and create a characteristic secondary beam of neutrinos. The beamline components are set on steel bedplates which measure $3.5^{\prime} \times 20^{\prime}$. Each bedplate rides upon a transporter which travels on a 30" narrow guage railway and can be linked together to form a train. Trains of up to ten units have been operated. This allows trains to be rapidly deployed to the Neutrino Target Hall (Neuhall) from the Target service Building (TSB) and vice-versa, via a railway system. The TSB has 
two work bays where trains may be serviced and two locked storage tunnels. The tunnels are covered by several feet of earth to protect the areas from potential tornado damage. This also serves as a radiation shield to prevent exposures to anyone outside the tunnels. Trains are stored there after an operating period so their induced radioactivity may safely decay. The trains which have operated in the Neutrino beamline are the Dichromatic, the Horn, the Triplet and the Sign selected Bare Target. The trains differ in their component configuration in order to produce secondary beams having different properties. A more detailed description of the Neutrino Area target and train systems can be found in a paper by Lindberg et al (Li75).

Target trains have been typically bombarded with 1 El 8 protons at $400 \mathrm{GeV}$ over a period of six months. This results in radioactivation of components which may exhibit residual radiation exposure rates of a few Roengten/hour $(R / h r)$ to tens of $R / h r$ at one foot from the source. These rates assume that several weeks of decay time have been allowed. Such is normally the case for routine maintenance operations.

Deposition of this much energy also inflicts damage to beamline components and instrumentation. Beryllium target pellets crack, motor drives fail, ion chamber wires disintegrate, brazed plumbing joints leak and electromagnets develop electrical ground faults. As a result, target trains often require hundreds of 
man-hours of routine maintenance after an operating period.

When Fermilab was designed in the late 1960's and early 1970's, it was anticipated that Neutrino target train servicing could be costly in terms of personnel radiation exposure. This was based in part on the expectation that target intensities of at least $1 E 13$ protons/pulse would be required to produce several neutrino interactions in a large bubble chamber detector. This was indeed later proven to be the case and historically the Neutrino beamline has been targeted with about one half of the protons available from the Main Ring. It was believed that much of the occupational radiation dose from the Neutrino Area could be spared by utilization of a remote manipulator system, which was eventually installed. It is the purpose of this report to examine the use of the Fermilab remote manipulator system and evaluate its cost effectiveness and success as an ALARA (As Low As Reasonably Achievable) tool.

Remote Manipulator System Description

The Fermilab manipulator system consists of two seperate units. The original system was a Mark IV electric master-slave manipulator developed at Argonne National Laboratory. The slave is mounted on an overhead bridge crane to allow a large work area in the TSB. The second system, called the Model M, was purchased 
from Central Research Labs of Red Wing, Minnesota. This system's slave unit is also mounted on a moveable bridge in the TSB. Both systems have their master units installed in a trailer 40 feet outside the radiation shielded TSB.

The two operating stations each have several high resolution television monitors for viewing the slave's work area. One technician controls the operation of the slave unit's two arms, as well as pan and tilt motions of the main television cameras. A second operator controls the manipulator and crane bridge motions, auxiliary television motions and all television zoom, focus and picture adjustments. These operations are controlled from a console behind the primary manipulator operator. A full description of the manipulator system, its performance specifications, and special tools can be found in a paper by simon et al (Si75).

\section{Method of Dose Estimates}

The manipulator system was employed most extensively in the period from 1977 to 1981. Personnel performing routine maintenance operations were monitored for radiation exposure incurred on the job by logging their daily pocket dosimetex readings. This allowed an accounting of the total dose equivalent incurred per operation. Each maintenance operation was reviewed 
to determine which subtasks had been performed using the manipulator system. This information was obtained from the manipulator operators logbook, interviews with the operations' supervisors and by direct observation of jobs by the author. An estimate was made for each such subtask to determine the manpower that would have been required to do the task manually. Estimates were made for several generic subtasks (eg. removal of a bolt, coolant hose disconnection, cable cutting) by timing technicians performing the task. Manpower estimates, i.e. number of person-minutes, were made for the remaining subtasks by Mechanical Support Group technicians who routinely do such work.

Records of residual radiation exposure rates on target train components were used in conjunction with the manpower estimates to determine the radiation dose equivalent spared by use of the manipulators. It was assumed that personnel performing a given task would incur doses at a rate equivalent to the measured radiation exposure rate one foot from the source. A summary of the dose equivalent spared for each major maintenance operation is shown in Figure 2. This figure also shows an estimate of the potential dose equivalent that would have been spared had all work been done remotely. The latter is simply a sum of the actual dose equivalent incurred plus the estimated dose equivalent spared by use of manipulators for each operation. 
The error in the cumulative dose equivalent incurred arises from two sources. The first is pocket dosimeter accuracy which is quoted as $\pm 10 \%$ by the vendor (Do82). The other error arises from accuracy of radiation exposure records. Persons involved in target train maintenance normally record their dosimeter reading whenever entering or leaving the work area. It is not unusual for workers to forget to $\log$ this information. It would be difficult to quantify this error, but it should be recognized that it has the effect of underestimating the dose incurred. The author's best judgement would be to assign an upper limit of $20 \%$ underestimation to the overall error.

The estimation of dose equivalent spared is subject to an even greater error. The first source is based on the estimate for the time required to perform a given task. The measurements and estimates made are conservative in that they assume that a given task is ideally executed. It makes no allowance for real-world occurances. Examples would include bolts that are frozen and will not come loose, extra time spent to recover dropped tools or equipment, and duplication of effort when an electrical or water connection is improperly made. Experience shows that these are common occurances. A realistic estimate of radiation dose spared must take this into account. 
The second source of error in dose equivalent spared is due to undocumented use of the manipulator system. Estimates of dose equivalent spared are based upon tasks which are known to have been performed. There have certainly been other occasions when the manipulators were utilized. Lapses in documentation and the memories of managers interviewed make it impossible to quantify the full extent of manipulator use. As such, the estimates made for dose equivalent spared can be considered lower limits. It is the author's best estimate that the true dose equivalent spared may be twice as high as the value reported in the next section.

Remote Manipulator System Usage Description

The term "routine maintenance" refers to the strip-down and replacement phases of target train refurbishment. The initial task in most of these remote refurbishment efforts has been to vacuum the bedplates and major components on the train. This is done to remove gross radioactive contamination such as rust, paint chips and dust. This greatly reduces the chance that any of the contamination could become airborne and thus preclude the need for respirators. It also minimizes the spread of contamination within the Target Service Building and any subsequent cleanup activity. Figure 3 shows the manipulator using a shop vacuum cleaner to remove contamination from a bedplate. The vacuum is mounted directly to the manipulator slave. The exhaust is fitted with a 
High Efficiency Particulate Air (HEPA) filter to prevent creation of ai rborne radioactivity.

The radionuclides involved are strictly beta and gamma emitters, typically at levels of tens to hundreds of nanocuries $/ 100 \mathrm{~cm}^{2}$. The principal isotopes found include Be-7, $\mathrm{V}-48, \mathrm{Mn}-54, \mathrm{Fe}-55, \mathrm{Co-57}, \mathrm{Co}-58$ and $\mathrm{zn}-65$. Many other isotopes as described in the classic text by Patterson and Thomas (Pa73) have been identified at Fermilab. However, most of those are produced in relatively small amounts or have half-lives less than a few hours. They are of little consequence during maintenance operations done following several days or weeks of decay time.

The second phase of remote maintenance on target trains typically involves removal of the few most radioactive components. Elimination of the several components with exposure rates above several $\mathrm{R} / \mathrm{hr}$ will generally leave the remaining exposure rates at a few hundred $\mathrm{mR} / \mathrm{hr}$. This latter level has been considered acceptable for manual maintenance tasks. Thus a train is usually remotely stripped of its target which substantially lowers the residual exposure rate on that bedplate. Figure 4 shows a manipulator operator at the controls of the master unit. He observes all activities via several high resolution television monitors and cameras mounted in the work area. Figure 5 shows a manipulator arm using a wrench to loosen a water coolant line from an aluminum target chill box. Figure 6 shows the disconnected 
line being grasped by the second manipulator arm. Once the coolant lines have been removed, the target and aluminum chill block may be unbolted from the bedplate and lifted from the train. Figure 7 shows an electric drill fitted with a socket wrench which can be used by the manipulator to remove bolts fastening the target. Also visible at left in Figure 7 is a beam position monitor called a segmented wire ionization chamber (SWIC). These devices are normaliy replaced as part of routine target train maintenance. This figure illustrates the confined work space of a target bedplate. A vacuum beampipe for a second beamline is visible in the foreground. Water and electric utilities run along the bedplate on the far side. Such obstructions are typical on target trains and hamper work operations, whether manual or remote.

often a magnet directly behind a target is damaged by radiation or is not expected to survive another operating period. Such magnets have been remotely replaced in several maintenance operations. Figures 8 and 9 demonstrate how the manipulator can attach a rigging hook and use its hoist to 1 ift a dipole magnet from its stand.

An examination of each major maintenance operation will allow a better understanding of the manipulator system usage pattern. The operations performed over a five year period are as follows: 
The Horn Train, so named for the shape of a large electrical conductor whose magnetic field focusses the secondary beam, required major refurbishment in 1977. Most of this work involved repair of leaking coolant water lines. This required brazing of plumbing joints for which the manipulators are impractical. As a result, technicians received a cumulative dose equivalent of 2.3 person-rem while the estimated dose equivalent spared is only 0.4 person-rem. This latter value represents 14.88 of the total dose equivalent ( 2.7 person-rem) that would have been spared had all work been performed remotely. In this case it was limitation of the manipulator system's ability that prevented greater usage.

The Triplet Train also underwent maintenance in 1977. This train utilized a quadrapole magnet focus-defocus-focus system to maximize the muon yield, hence the name Triplet. The remotely performed work was limited to the target bedplate. It included removal and installation of SWICs and a target drive system. An attempt to install a beam collimator failed because the manipulator cameras could not view the guide pins. In this case it was incompatability of component design with the manipulator system that stymied the effort. However, it is likely that more extensive use of the manipulators could have been made in this operation. A cumulative dose equivalent of 5.5 person-rem was incurred versus 0.7 person-rem spared. The latter figure represents $11.3 \%$ of the 6.2 person-rem total that could have been spared had all work been performed remotely. 
The Dichromatic Train produces a sign and momentum selected beam of mesons that decay into muons and neutrinos. The train is so named because the decay neutrinos are produced with two distinct momentum peaks. Work done on the Dichromatic Train during the summer of 1977 consisted of stripping old components from the bedplates. A cumulative dose equivalent of 0.7 person-rem was incurred versus 1.2 person-rem spared. The latter figure represents 63.18 of the 1.9 person-rem total that could have been spared had all work been performed remotely.

The decision to operate the Horn Train in early 1979 was made shortly before the run period. Work in progress on the Dichromatic and Triplet Trains was dropped and all efforts were shifted to prepare the Horn. As a result there was no time afforded for remote work. A cumulative dose equivalent of 1.7 person-rem was incurred. Here an unforeseen change in the physics research program prevented use of the manipulator system.

The 1979 Dichromatic Train maintenance employed the manipulators in a unique fashion. A caulking gun was used to apply a thermoconductive paste to a pair of dipole magnets which had experienced overheating. Water cooled heat exchange panels were then installed on these surfaces. The only part of this task done manually was the final banding of the panels to the magnets. Another successful remotely performed task was the exchange of a cracked berylium target pellet for a new one. Figure 10 shows the 
manipulator removing a keeper plate from the aluminum target pellet holder. This water cooled holder was then tilted as shown in Figure 11 to allow the $1 "$ diameter berylium pellets to slide out. It should be pointed out that most of the photographs shown in this paper were staged using components having little or no residual radioactivity.

The cumulative dose equivalent incurred for this operation was 2.6 person-rem. It is estimated that 1.1 person-rem was spared which is 29.78 of the 3.7 person-rem total that would have been spared had all work been done remotely.

The Triplet Train required relatively minor refurbishment in late 1979, with most work done in the area of the target. The manipulators were used to remove and replace target pellets, remove a sWIC and place lead shielding blankets on a highly radioactive component. This latter task was done to attenuate the gamma exposure rate at an adjacent magnet which required manual brazing of water lines.

The cumulative dose equivalent incurred was 1.3 person-rem versus 0.7 person-rem spared. The latter represents $35 \%$ of the total dose equivalent that could have been spared if all work had been done remotely. 
The Horn Train operation of late 1979 and early 1980 implemented a new design which called for the decomissioning of almost half its bedplates. Many of the components were remotely removed and disposed of as radioactive waste. The target and its drive were removed and replaced remotely. A beam dump and a magnet were also removed from this train.

The manipulator system was also used to vacuum gross contamination. The Horn Train in particular would accumulate a heavy layer of rust during a beam operating period. All trains during the era of Main Ring operation were targeted within a six foot diameter by 200 foot long iron pipe called the target tube. The Horn Train's electrical conductors would be pulsed with a current of $140 \mathrm{kiloAmps}$ at $7 \mathrm{kilovolts}$ to produce a $100 \mathrm{kilogauss}$ magnetic field. This was done to focus the secondary beam particles produced after targeting the primary proton beam (Gr75). Although the precise mechanism is not understood, pulsing the Horn Train in this manner causes the iron target tube to vibrate with sufficient amplitude that it shakes loose rust scales. This occurs even though the target tube is buried beneath 26 feet of earth.

This maintenance operation incurred a cumulative dose equivalent of 6.0 person-rem. The 1.5 person-rem spared is $20 \%$ of the total dose equivalent that could have been spared if all work had been performed remotely. Much of this work involved brazing 
new water connections, a task for which the manipulator system is not suited.

The final operation examined is the Dichromatic Train maintenance of 1981. The manipulator system was employed for work on the target bedplate while all other areas were serviced manually. This policy was adopted because the residual radiation exposure rates were very high at the target bedplate (about 2 $\mathrm{R} / \mathrm{hr}$ ) but only moderately high (typically $100 \mathrm{mR} / \mathrm{hr}$ ) at the nine other bedplates. This type of decision applied to many of the earlier maintenance operations, i.e. use the manipulators in the few high exposure rate areas but do the work manually in all other areas. Reasons for this practice are examined in the Discussion section.

The cumulative dose equivalent incurred during this operation was 3.3 person-rem. The 1.0 person-rem spared is $23.3 \%$ of the 4.3 person-rem total that would have been spared had all work been done remotely.

Overall, the total dose equivalent received during this five year period in performance of routine train maintenance is 23.4 person-rem. It is estimated that utilization of the manipulators has spared 6.6 person-rem. This savings represents $22 \%$ of the 30 person-rem that could potentially have been spared if all target train maintenance had been performed remotely. 


\section{Discussion}

Several of the factors which prevented greater use of the manipulator system and a further reduction in cumulative dose equivalent are identified in the previous section. These include limitations in the manipulators' ability to execute certain tasks, train component design incompatible with manipulator abilities, and time constraints caused by unforseen changes in the physics research program. However, the principal reason for actual dose spared being a small fraction of the potential is that the system was generally employed in only the few high exposure rate areas. The dose equivalents actually incurred were due to many man-hours of manual work in areas of moderate exposure rate. This policy has been common because it has been concluded by persons involved with these systems that a task which takes one man-hour to do manually requires eight man-hours to accomplish remotely (Gr81). This conclusion is based on the operational experience of several managers, engineers and support group technicians who have worked with these systems over a period of years. There is also support for this conclusion based on a study performed for NASA by Argonne National Laboratory. This study measured the time required for various mechanical tasks performed manually as well as with manipulator systems similar to those at Fermilab. A task time ratio of 9:1 for remote versus direct manual work was measured during initial attempts by the study group. Improved tooling and practice allowed this ratio to be reduced to $3.7: 1$ (ANL67). 
In view of these conclusions, the managers have generally not considered it ALARA effective to employ the manipulators in any but the highest radiation exposure rate circumstances. Constrained time schedules have often left little choice but to utilize the system where it would be most ALARA effective.

There are many factors which contribute to this ratio of $8: 1$ for time to do tasks remotely versus manually. These include lack of depth perception afforded the manipulator operator viewing television monitors, poor gripping ability of manipulator tongs for certain tools, inability to view the work area, inability to reach the work area, delays due to system malfunction and low operator proficiency at utilizing the system.

Most of these problems could be overcome or significantly reduced if a modest level of manpower was dedicated for continued development of the manipulator systems. However, during the time period examined, this has not been the case. The manipulator system has instead been utilized sporadically by persons having other primary duties. It has often been totally neglected for periods of several months and then called upon to perform complicated tasks on a few days notice. The results have frequently been frustrating to those involved and have prevented more extensive use of the system beyond the highest radiation exposure rate areas of any target train. 
This should not be seen as a criticism of those responsible for the manipulator system. As stated earlier, this group has other primary responsibilities which are of a critical path nature. Tight operating schedules have frequently involved all available personnel on an overtime basis. It is no wonder that the manipulator system has been neglected until situations arose that demanded it use. Seen in this context, the author believes that the pattern of manipulator system usage has reflected the principle of ALARA. That is, in this two tier exposure rate circumstance, the system has generally been employed where it would be most effective. Within the constraints of an $8: 1$ time trade-off for remote versus manual work and the short schedules for completion of work, it would not have been reasonable (nor often possible) to use the system in the lower level of the tier. A significant reduction in this $8: 1$ time ratio would be necessary before widespread use of the manipulator system would become feasible.

The 8:1 time ratio could certainly be reduced by several means. Assignment of an electro-mechanical equipment specialist on even a part-time basis to maintain the system would reduce time lost due to breakdowns. Specific personnel could be designated as manipulator operators and be scheduled proficiency exercises on the system at least several hours a week. This would sharpen their skills and maintain each operator's ability to perform efficiently. Weekly exercises would also allow early detection of 
any malfunctions in the manipulator system. Further reductions in lost time could be made if engineering manpower was dedicated to development of special tools and tool grips that would allow the manipulator to better grasp and employ such equipment. Operators frequently lose time when a tool is dropped by the manipulator slave and must be retrieved. It would not likely be feasible for Fermilab to attempt improvements in the present television viewing system. That is, two dimensional images may have to be acceptable until improved technology is available from an outside source.

The cost effectiveness of the manipulator system as an ALARA tool can be measured by assessing the operating and capital equipment costs versus dose equivalent spared. Since no full time manpower was ever dedicated for the manipulator system nor any accounting made of part-time efforts, only a rough estimate can be provided for the operating costs.

It is known that seven major maintenance operations utilized the manipulator system during the five year period examined. It is estimated that two senior and two junior technicians worked full time with the manipulators for three weeks per maintenance operation. The 1982 median pay scale for these employee categories is $\$ 558 /$ week and $\$ 337 /$ week, respectively, and Fermilab costs for their benefits is estimated as an additional 20\% (Ju84). Thus the operating cost can be calculated as: $2(\$ 558+\$ 337) / \mathrm{wk} \times 1.2 \times 3 \mathrm{wk} /$ operation $\times 7$ operations $=\$ 45 \mathrm{~K}$ 
This figure is in 1982 dollars and covers all operations performed from 1977 through 1981 .

The Mark IV manipulator system was surplused by Argonne National Laboratory and obtained free of charge by Fermilab in 1970. The Model M systen was purchased from Central Research Labs in 1975 for $\$ 135 \mathrm{~K}$. Additional expenditures for the bridge and turret mounting system, television cameras and monitors and controls electronics brought the total cost to $\$ 250 \mathrm{~K}$ in 1975 dollars. This system was estimated by the vendor to cost $\$ 400 \mathrm{~K}$ if purchased in 1982 (Je82). The manipulator systems can be periodically upgraded and are considered to have a 20 year lifetime before becoming obsolete.

Amortized over this 20 year period, the capital cost for the five year period examined is \$100K in 1982 dollars. Combined with the estimated operating cost, the total cost for the working system is $\$ 145 \mathrm{~K}$ for the five year period.

Therefore, it can be estimated that the 6.6 person-rem spared was at an economic cost of $\$ 22 \mathrm{~K} /$ person-rem. This should be taken as an upper limit since the estimated dose equivalent spared is a very conservative value. If, as discussed earlier, the true value is 13.2 person-rem, then the cost per person-rem spared may be as low as $\$ 11 \mathrm{~K}$. 
The application of the ALARA philosophy requires a judgement of what is considered reasonable. Several scientific and government bodies have offered guidance in this matter and it would be appropriate to review this here. Fermilab is regulated and primarily funded by the United States Department of Energy. This agency has provided guidance which states, "if dose reduction can be achieved at a cost of less than or equal to $\$ 2000$ per person-rem, then it is cost-beneficial and should always be done" (Ka80). This reference further suggests that a reasonable upper limit on expenditures to spare a person-rem would be $\$ 60 \mathrm{~K}$. Applying a $7 \%$ annual inflation rate, these figures scale to $\$ 2.3 \mathrm{~K}$ and $\$ 68.7 \mathrm{~K}$, respectively in 1982 dollars. Based on this range, the figures of $\$ 11 \mathrm{~K}$ to $\$ 22 \mathrm{~K}$ determined for the remote manipulator system would appear reasonable. However, several other sources of guidance would be in disagreement with this conclusion.

The U.S. Nuclear Regulatory Commission has justified expenditures of $\$ 1000$ per person-rem for control measures (CFR75). This value scales to $\$ 1.6 \mathrm{~K}$ in 1982 dollars. The National Academy of Sciences Committee on the Biological Effects of Ionizing Radiation has suggested a range of $\$ 12$ to $\$ 120$ per person-rem (BEIR72). This scales to a range of $\$ 24$ to $\$ 240$ per person-rem in 1982 dollars. The International Commission on Radiological Protection has suggested a range of $\$ 10$ to $\$ 250$ per person-rem which scales to an $\$ 18-\$ 460$ range in 1982 dollars (ICRP73). 
Clearly, the estimated expenditures by Fermilab to reduce personnel radiation dose equivalents far exceeds the ranges suggested by these three latter sources. It is the author's opinion that a value of $\$ 2000$ per person-rem is a reasonable expenditure for a dose reduction program. In general, I would not consider it ALARA effective to spend in excess of $\$ 10 \mathrm{~K}$ to spare a person-rem of dose equivalent. Thus, when viewed strictly in terms of dose spared per dollar. I would conclude that the manipulator system has been at best marginally successful as an ALARA tool.

However, it would be an oversimplification to evaluate the remote manipulator system solely on ALARA effectiveness. This evaluation was based on experience with scheduled maintenance operations. Occasionally unplanned failures occur and the manipulator system has been employed to effect repairs. The most notable failure took place in December 1976 and involved the Triplet Train.

At that time the proton target intensity was raised to as high as 2 E13 protons/pulse while the extraction cycle was reduced to 7 seconds. This yielded a beam power about three times greater than had been run during the previous six months. This Triplet Train employed an eight foot long steel beam collimator directly downstream of the target to intercept the spray of secondary particles which would otherwise impact a quadrapole magnet. The 
increased power caused the collimator to overheat. Since the collimator support stands were located near it's ends, it began to sag in the middle. This caused it to intercept part of the primary proton beam which further aggravated the situation. Eventually the collimator failure was detected and the Triplet Train was removed from the target tube. A residual radiation survey done after a one week decay period found levels of $200 \mathrm{R} / \mathrm{hr}$ at one foot from the collimator. Contamination levels on the train were up to $2000 \mathrm{nCi} / 100 \mathrm{~cm}^{2}$ (Go77).

The train was brought to the target service building where remote repairs began. First the train was vacuumed to remove gross radioactive contamination. The collimator was then remotely unbolted with a pneumatic wrench. It was then rigged and lifted from the bedplate by the manipulator and an overhead crane. The collimator stands, target and target drive were also removed remotely and discarded as radioactive waste. Once cleared of these components, residual radiation exposure levels were low enough to permit manual refurbishment.

In this case manual repairs would not have been possible. An object with a gamma radiation exposure rate of $200 \mathrm{R} / \mathrm{hr}$ cannot be directly approached by a technician. The use of portable radiation shielding is generally impractical for train work. The need to reach over utilities and into tight spaces from beside the bedplate makes a shield wall. with arm portals too restrictive. It 
is also not feasible to cover such a highly radioactive component with lead shielding. The personnel radiation exposures in doing so would be prohibitively high. Without the manipulator system, the only alternative would be to wait for the object to decay to a low enough radiation level to permit manual work. A steel component like the Triplet collimator, measuring $200 \mathrm{R} / \mathrm{hr}$ one week after a several day irradiation period, would require an additional 10 weeks of "cooloff" to decay to an approachable level of about $10 \mathrm{R} / \mathrm{hr}$. This estimate is based upon calculations by Barbier of induced radioactivity and decay curves for iron (Ba69).

In the case of the Triplet train failure, the Sign Selected Bare Target train was substituted. This was considered tolerable since the highest priority experiment operating at that time also required an SSBT run as part of its schedule. Other lower priority experiments, however, suffered a loss of necessary data. Most neutrino beamline experiments conducted in a given operating period require use of a specific train. Beam running time is carefully budgeted and is difficult to recoup. If a given train fails and a decay period is necessary prior to repair work, the cost of this delay must be considered.

While it would be difficult to assign a dollar value to a particular high energy physics experiment, other operating expenses are well known. Electrical power costs to operate the Main Ring during 1982 were $\$ 2000$ per hour (Ge82). The neutrino 
trains would typically target half of the protons available from the accelerator. It can be argued that an inability to use $50 \%$ of the protons accelerated represents a waste of electrical power at the rate of $\$ 1000$ per hour. Assuming a normal operating duty cycle of 0.7 , a one week loss of a target train represents an economic loss of $\$ 118 \mathrm{~K}$ and a one month loss would be $\$ 504 \mathrm{~K}$.

\section{Summary and Conclusion}

Fermilab's experience with a remote manipulator system indicates that it has spared a cumulative occupational radiation dose equivalent of at least 6.6 person-rem during the five year period of 1977-1981. The true value for dose equivalent spared may actually be a factor of two higher. The group which utilized the system incurred about 23.4 person-rem in target train maintenance during this period. Thus the dose equivalent spared represents 228 of the potential dose equivalent that could have been spared had all train work been done remotely.

It is concluded that the persons utilizing the manipulator system generally applied good ALARA principles. The system was employed in seven of the eight major operations studied and a significant fraction of the potential cumulative dose equivalent was avoided. Any more extensive use of the system would have spared personnel radiation doses at much greater expense in time 
and manpower.

The estimated cost in sparing radiation dose equivalent is shown to be no greater than $\$ 22 \mathrm{~K}$ per person-rem and perhaps as low as $\$ 11 \mathrm{~K}$ per person-rem. It is concluded that the manipulator system is an expensive means of reducing radiation dose to employees. As such, it is judged to be only marginally effective as an ALARA tool. However, based on a cost versus cost comparison the manipulator system can easily be justified.

The system has demonstrated its ability to make emergency repairs that would otherwise not be feasible. The economic value of beam time which the system can spare in a single emergency operation could easily underwrite its entire capital investment. As such, the remote manipulator system can be seen as a worthwhile investment. 
Acknowledgements

The author would like to thank Don Carpenter, Norm Gelfand, John Grimson, Jack Lindberg and Ray Stefanski for the invaluable information they provided on Fermilab beamline and manipulator systems operating experience. I also wish to thank Don Carpenter, Mark O'Malley, Lee Simmons and Rick Fenner for their assistance in the photography sessions, Harry Ferguson III and Graciela Finstrom for the figure artwork and Borys Jurkiw for assistance in cost estimates. Thanks also to J.D.Cossairt and Jack Lindberg for reviewing this paper and providing many valuable suggestions. I especially wish to thank my wife Carol for her support throughout this project. 


\section{REFERENCES}

ANL67 Argonne National Laboratory, 1967, Manipulator systems for Space Application, Volume II Technical Report, Manned Space Flight study No. 981-10-30-04, pp. 63-73, Argonne National Laboratory, 9700 South Cass Ave., Argonne, IL 60439.

Ba69 Barbier M., 1969, Induced Radioactivity, p. 344, Fig. B.14 (Amsterdam: North-Holland Publishing Company) (New York: John Wiley \& Sons, Inc.).

BEIR72 Advisory Committee on the Biological Effects of Ionizing Radiation, 1972, The Effects on Populations of Exposure to Low Levels of Ionizing Radiation (Springfield, VA: National Technical Information Service).

CFR75 Code of Federal Regulations, 1975, "Numerical Guides for Design Objectives and Limiting Conditions for Operation to meet the Criterion 'As Low as is Reasonably Achievable' for Radioactive Material in Light-Water-Cooled Nuclear Power Reactor Effluents", 10 CFR 50, Appendix I.

Do82 Dosimeter Corporation, 1982, Direct Reading Gamma and $\mathrm{X}$-Ray Dosimeters - Specifications, (Cincinnati, OH).

Ge82 Gelfand N., 1982, Personal Communication, Director's Office, Fermi National Accelerator Laboratory, Batavia, IL 60510.

Go77 Gollon P.J., 1977, Interim Report on Neutrino Collimator Destruction and Neuhall Contamination - December 28-29, 1976, Internal Memo, Fermi National Accelerator Laboratory, Batavia, I1 60510 .

Gr75 Grimson J., Lindberg J., Simon J. and Theriot D., 1975, Design of the Fermilab Neutrino Horn Train Referenced to Radioactive Maintenance", in: Proc. of 23rd Conf. on Remote Syst. Technol., 24.

Gr81 Grimson J., 1981, "Applications of Robotics in Accelerator Research Facilities", Paper presented at Workshop to Delineate the Economic, Technical and Policy Issues for Remote Maintenance in Energy Systems, 9-10 March, University of Florida, Gainesville Florida.

ICRP73 International Commission on Radiological Protection, 1973, "Implications of Commission Recommendations that Doses be Kept as Low as Readily Achievable", ICRP publication 22 (Oxford: Pergamon Press).

Je82 Jelatis D.G., 1982, Personal Communication, Central Research Laboratories Division, Sargent Industries, Red Wing, MN 55066. 
Ju84 Jurkiw B., 1984, Personal Communication, Personnel office, Fermi National Accelerator Laboratory, Batavia, IL 60510.

Ka80 Kathren R.L., Selby J.M. and Vallario E.J., 1980, A Guide to Reducing Radiation Exposure to As Low As Reasonably Achievable (ALARA), U.S. Department of Energy, DOE/EV/1830-T5.

Li75 Lindberg J., Grimson J., Simon J. and Theriot D., 1975, "Fermilab Target Areas and Target Train Systems", in: Proc. 23rd Conf. Remote Syst. Technol., 41.

Pa73 Patterson H.W. and Thomas R.H., 1973, Accelerator Health Physics, pp. 503-531 (New York: Academic Press).

Si75 Simon J., Grimson J., Lindberg J. and Theriot D., 1975, "Design of the Fermilab Remote Target Maintenance System", in: Proc. 23rd Conf. Remote Syst. Technol., 41. 


\section{LIST OF FIGURE CAPTIONS}

1. Plan view of Fermilab and Neutrino target area.

2. Actual and potential dose equivalent spared during proton target train maintenance.

3. Manipulator slave using vacuum cleaner to remove radioactive contamination.

4. Manipulator operator at master control unit.

5. Manipulator slave unit using ratchet wrench to loosen target chill block's cooling water line.

6. Target chill block's cooling water line is removed by manipulator slave.

7. Manipulator slave with electric socket wrench, preparing to unbolt target chill block from stand.

8. Manipulator slave attaches overhead crane's lifting sling to a small dipole magnet.

9. Manipulator slave's overhead crane lifts small dipole magnet from it's stand.

10. Manipulator slave removes target pellet keeper plate from chill block.

11. Manipulator slave tips target chill block to allow beryllium target pellets to slide out and be replaced. 
Figure 1 PLAN VIEW OF FERMILAB AND NEUTRINO TARGET AREA

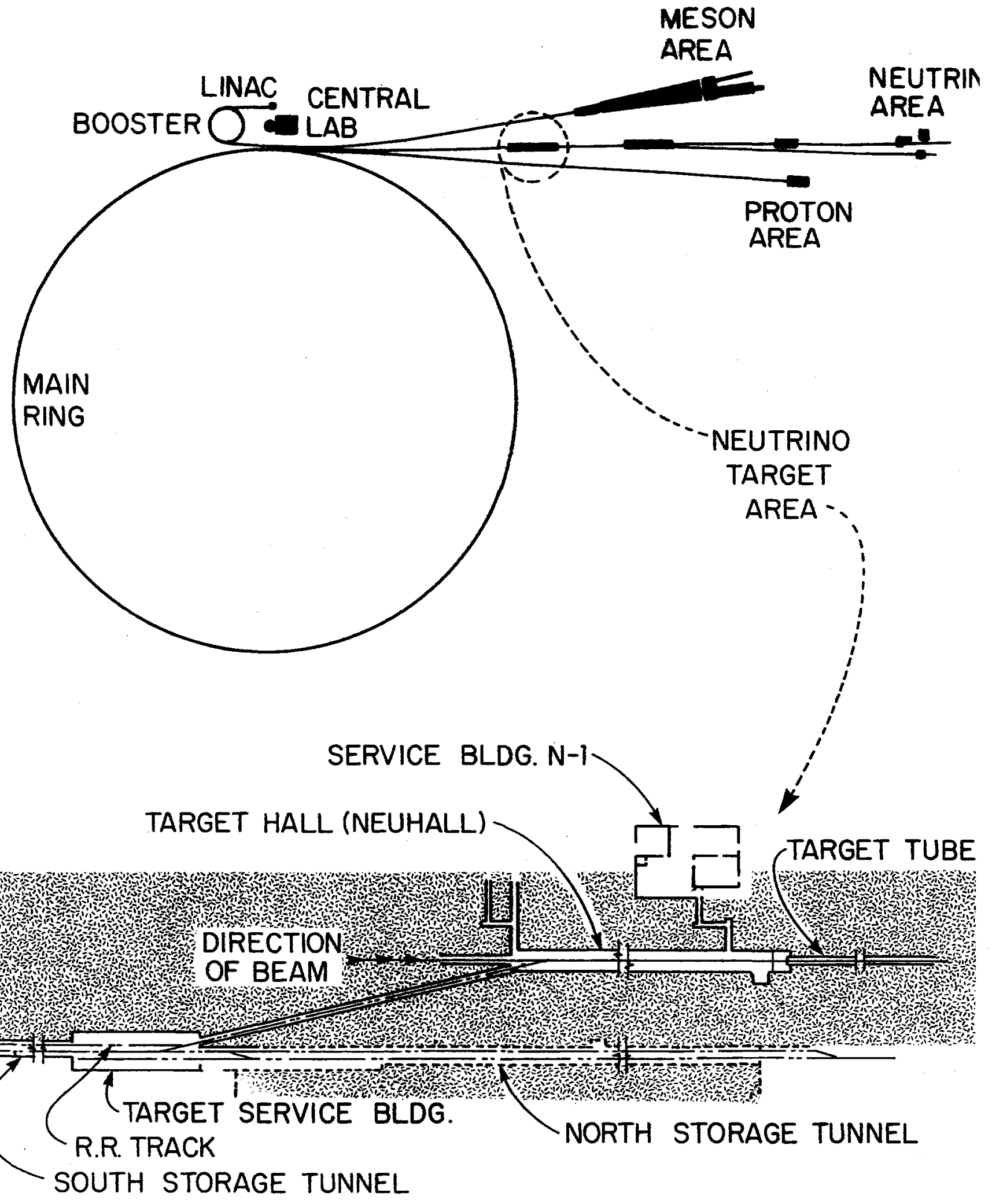




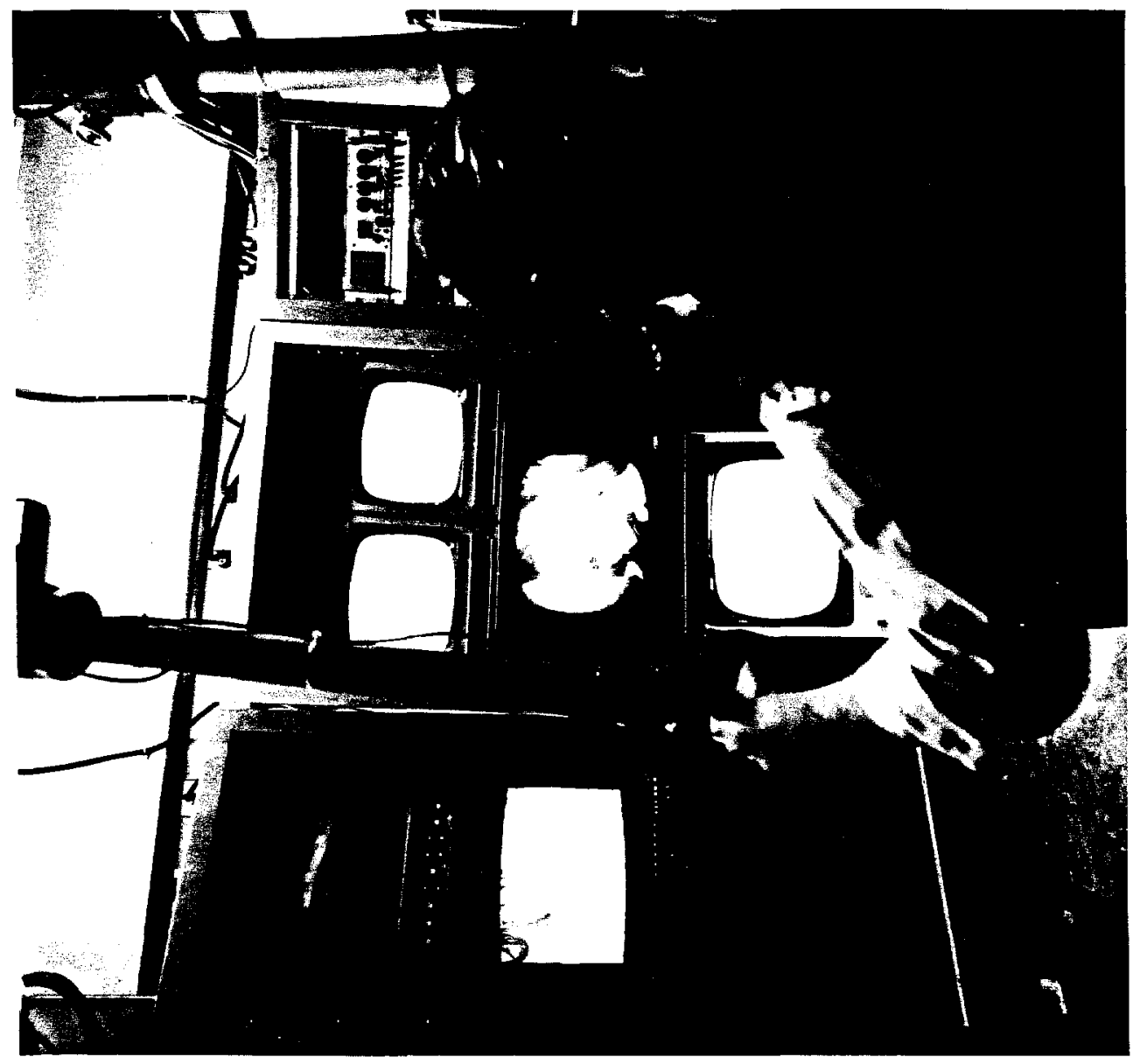

\begin{tabular}{l}
$\square$ \\
0 \\
5 \\
$\vdots$ \\
\hdashline
\end{tabular}

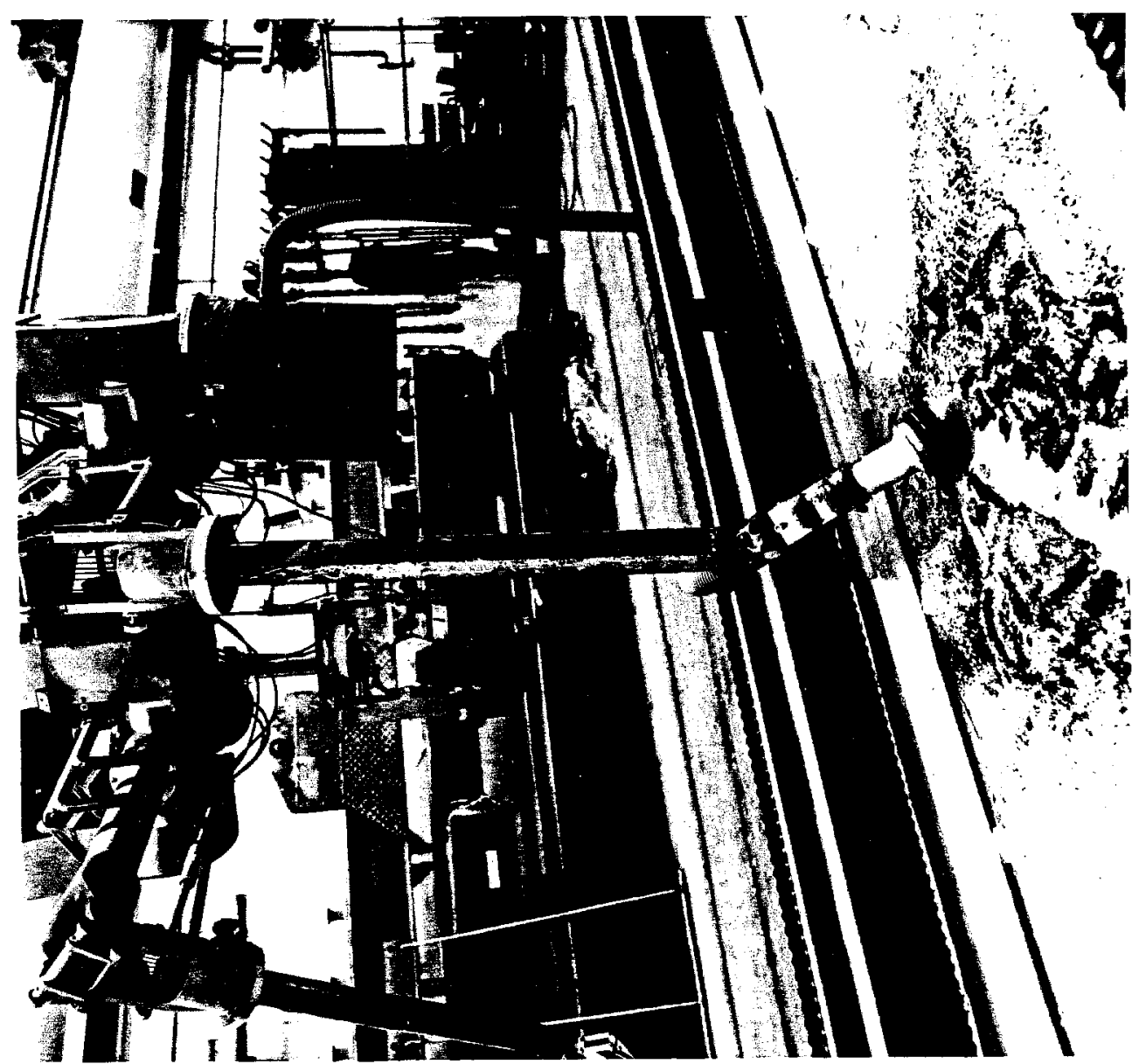

$m$
0
$\frac{5}{3}$
$\frac{0}{4}$ 

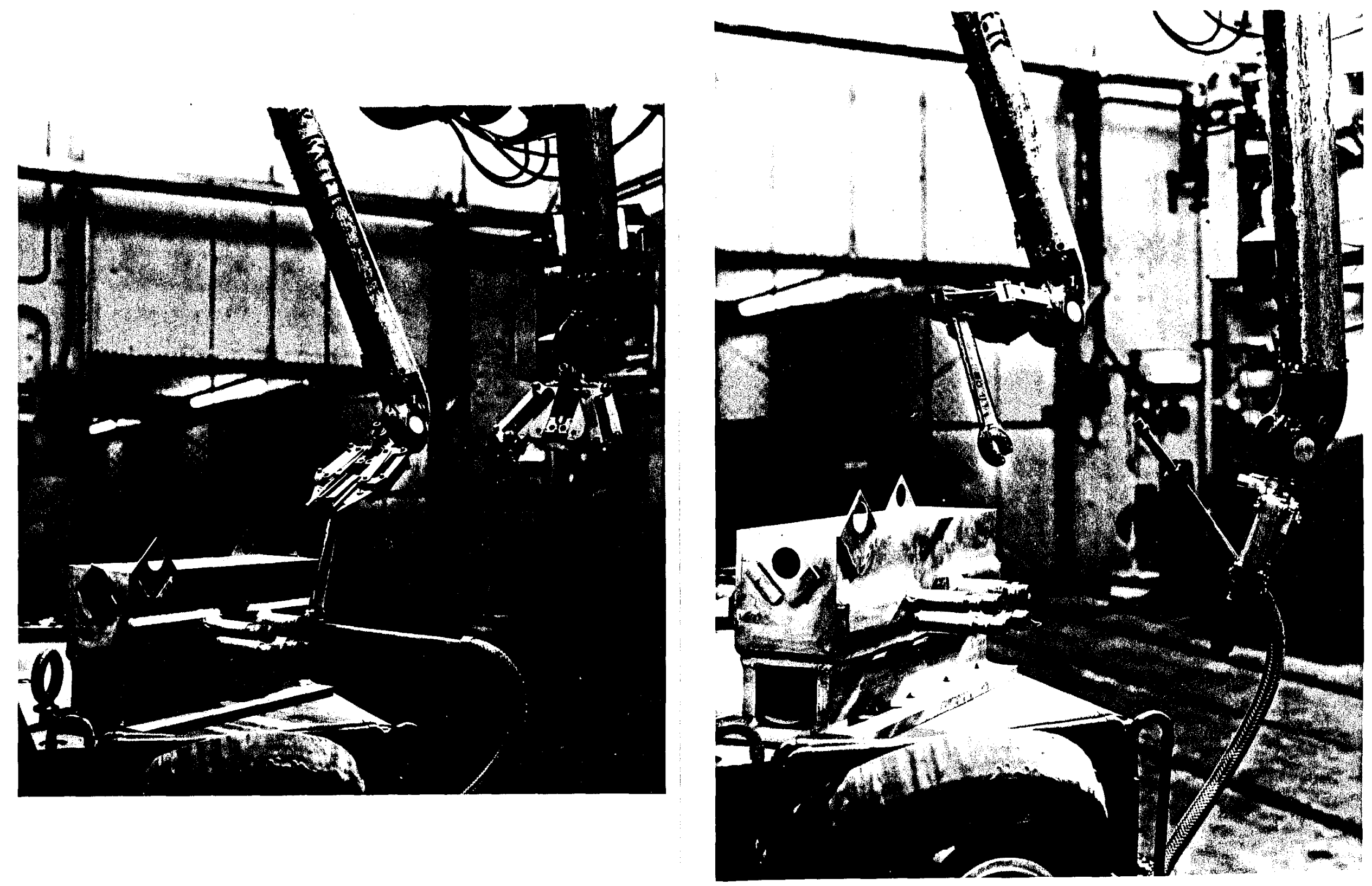

Figure 5

Figure 6 


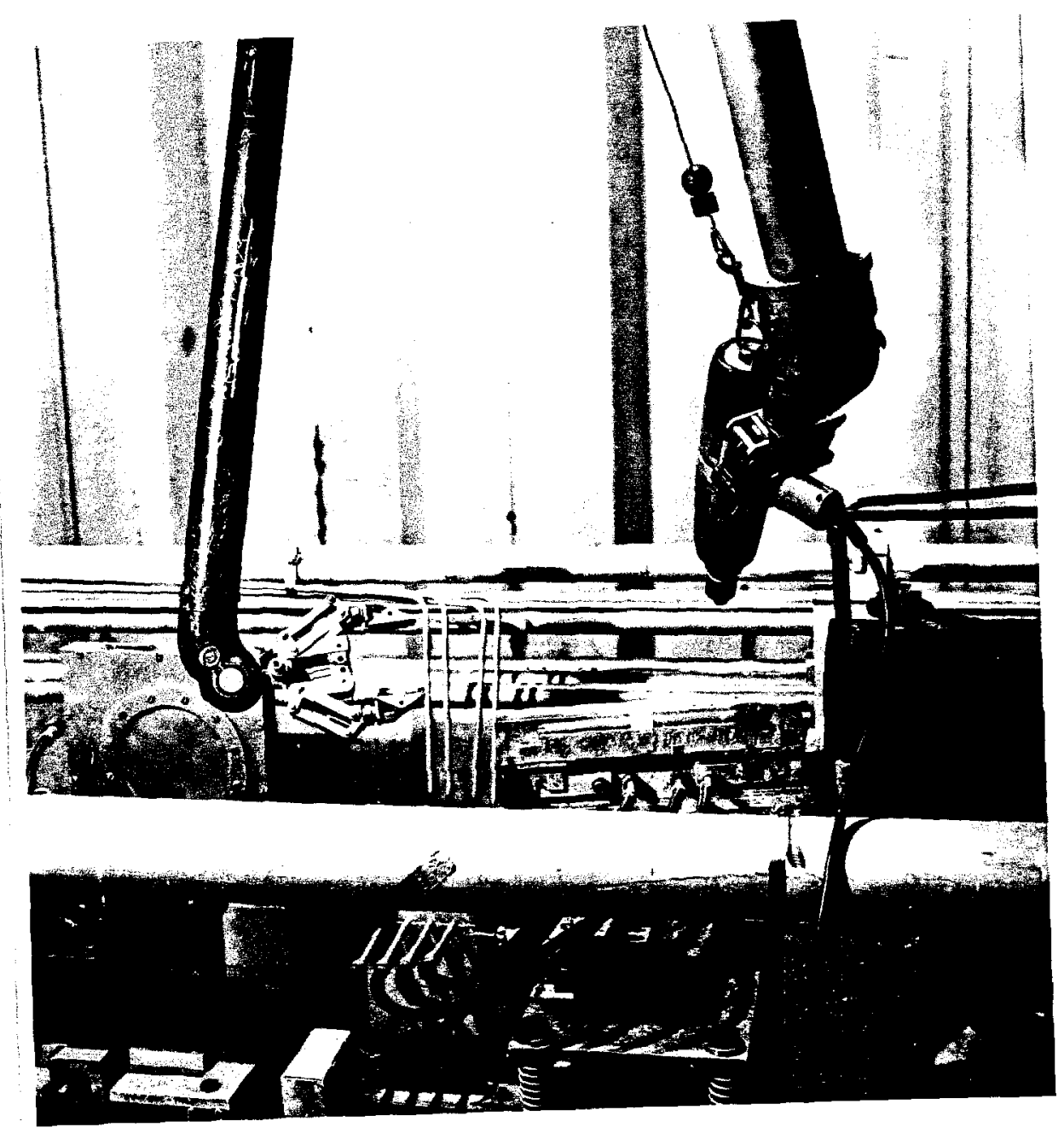

Figure 7 


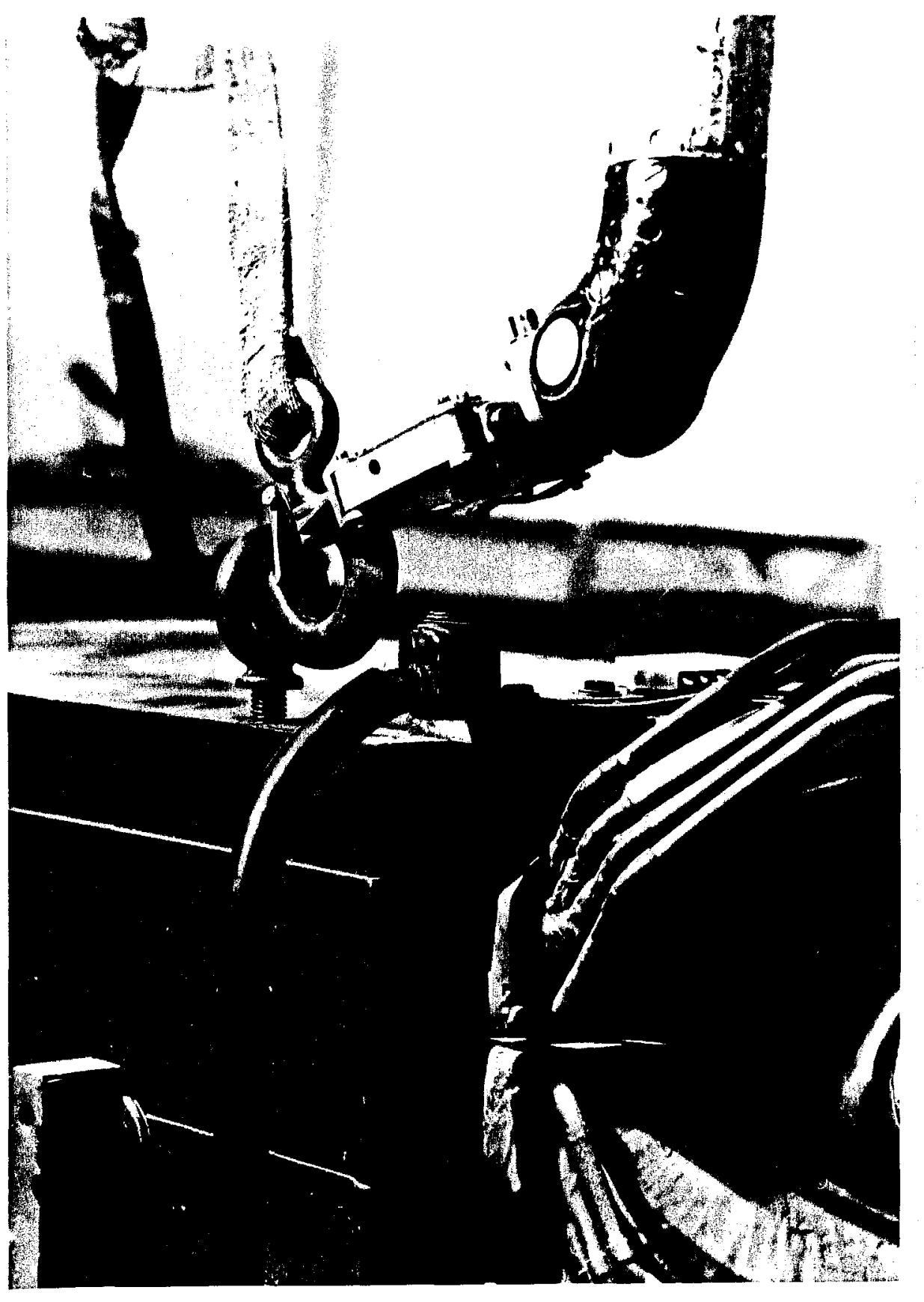

Figure 8

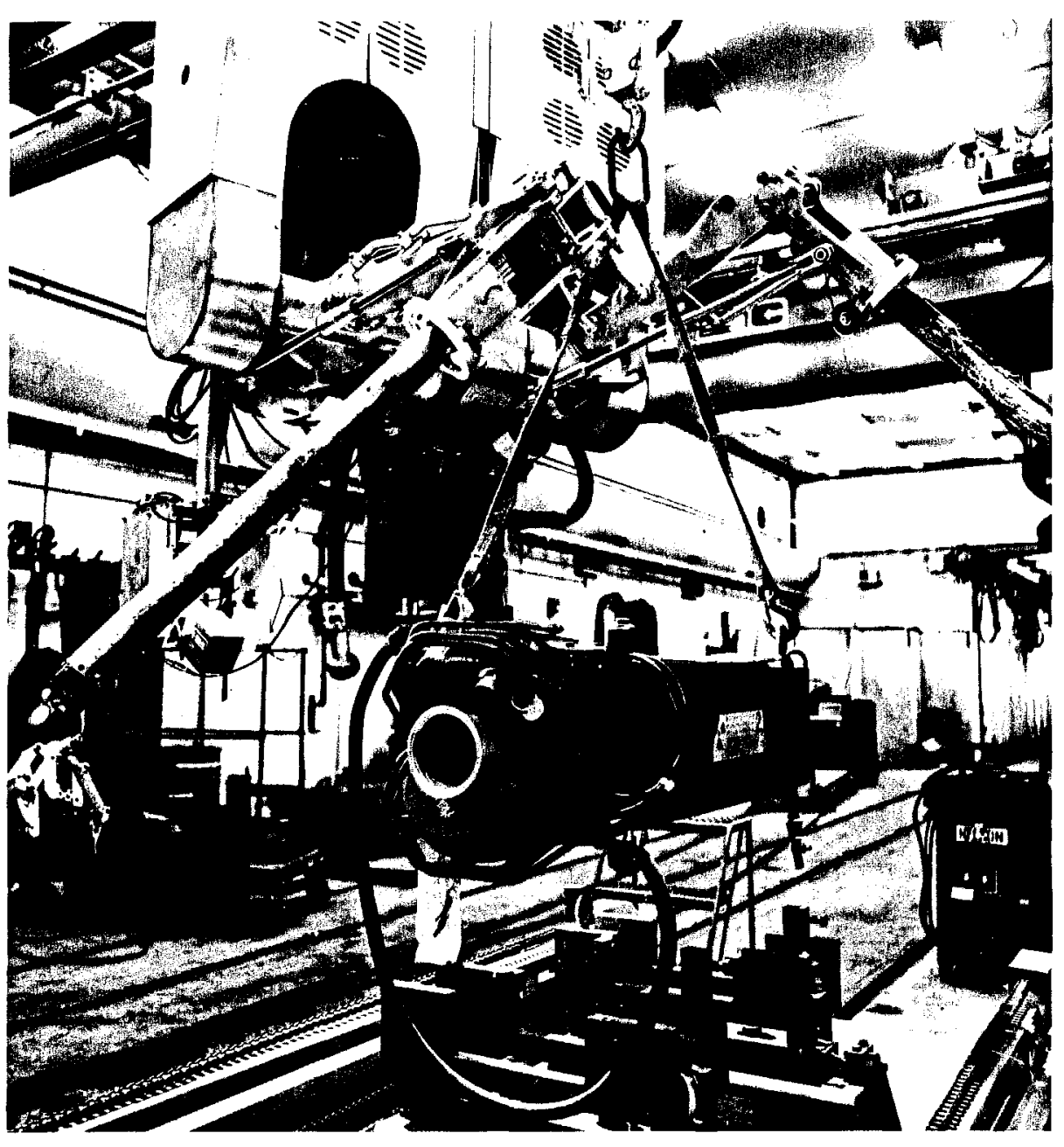




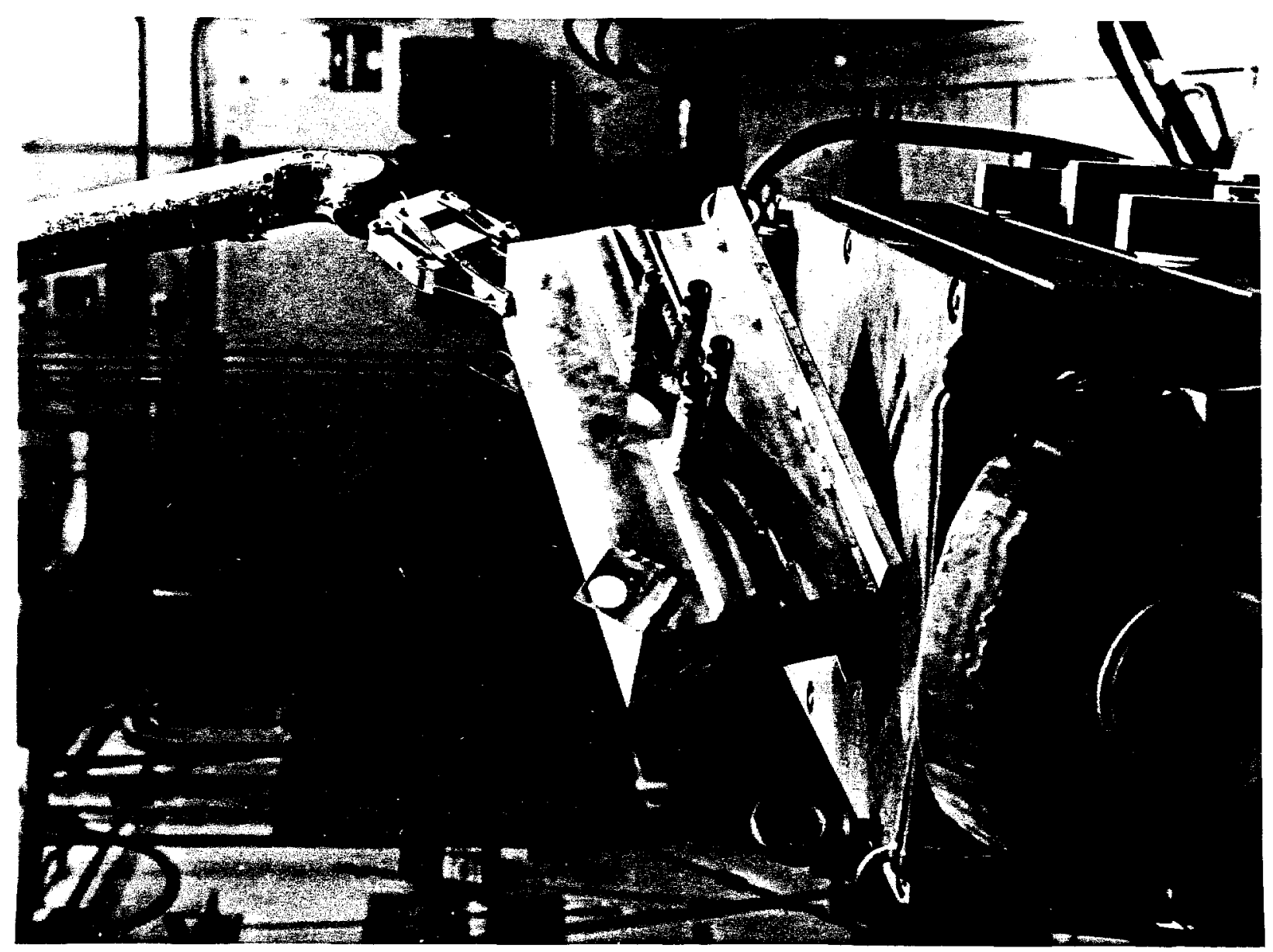

-
0
몬
노

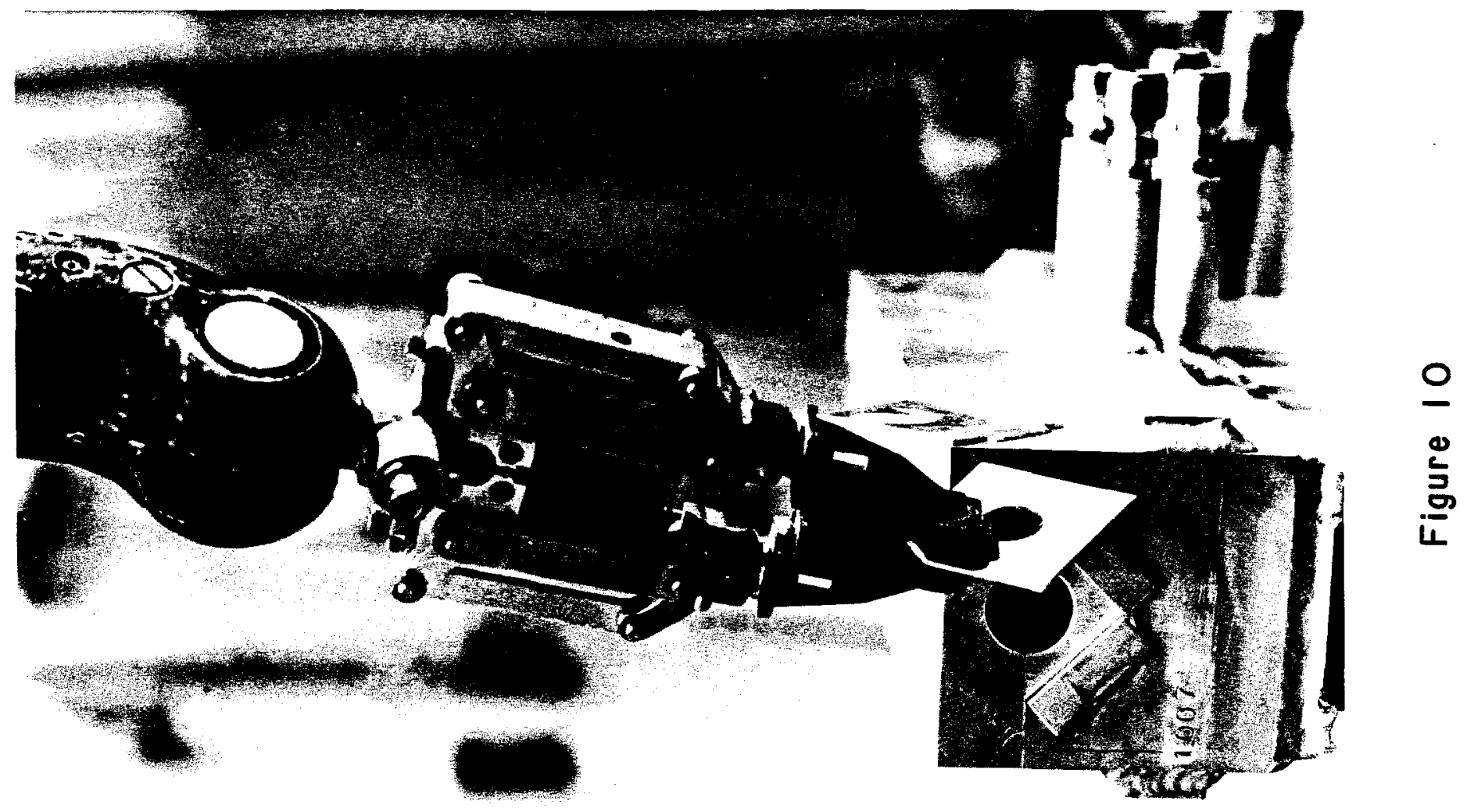

